\title{
LA CHUTE DES GÉANTS : DE LA CRISE À L'ÉMERGENCE
}

\author{
Author(s) / Auteur(s) : \\ Prof. Damien CLAEYS \\ Faculté d'architecture, d'ingénierie architecturale, \\ d'urbanisme (LOCI) \\ Laboratoire Théorie des systèmes en architecture (tsa-lab) \\ Université catholique de Louvain (UCLouvain) \\ damien.claeys@uclouvain.be
}

\author{
Claude LAMBERT \\ Informaticien, Systémicien \\ Vice-président de l'Union européenne de systémique (UES) \\ Président de l'asbl Systèmes \& Organisations $(S \& O)$ \\ cepehello@gmail.com
}

\begin{abstract}
Résumé :
Peinte par Jules Romain au palais du Té de Mantoue, la fresque appelée La chute des Géants possède une force suggestive opérante à plusieurs niveaux permettant un questionnement original du concept multiforme de crise. L'inscription de cette fresque dans son contexte historique et un intermède mythologique montrent que le thème développé dans cette æuvre allégorique éclaire l'état actuel de notre civilisation, affectée d'un crash imminent, à la fois, écologique, sociétal et humain.

Avec la méthode analytique, la gestion de la crise consiste à limiter la croissance à cause de la rareté des ressources naturelles disponibles. Mais avec la théorie des systèmes, lors de processus de transition de phase, le concept de seuil semble être directement lié à celui d'émergence. Pour modéliser des crises, les concepts de limite et de croissance pourraient donc avantageusement être remplacés par ceux de seuil et d'émergence.

À travers ces distinctions à priori théoriques, la communauté des systémiciens auraient un rôle pratique à jouer pour tenter de sortir de la crise de manière alternative et redonner un nouvel horizon.
\end{abstract}

\section{Keywords / Mots-clés :}

crise, seuil, émergence, décroissance, autonomie, titan, géant, Mantoue, palais du Té, Romain, Gonzague, chute

Aviez-vous remarqué l'illustration centrale de l'affiche du congrès UES2018. Elle présente un détail de la fresque appelée La chute des Géants peinte par Jules Romain au palais du Té.

Mais pourquoi choisir cette fresque emblématique du maniérisme italien?

Après l'inscription de celle-ci dans son contexte historique et un intermède mythologique, nous interpréterons la signification qu'elle semble exprimer de manière détournée et nous montrerons que le thème développé dans cette œuvre allégorique éclaire l'état actuel de notre civilisation, affectée d'un crash imminent, à la fois, écologique, sociétal et humain.

\section{CONTEXTE D'ÉLABORATION DE LA FRESQUE}

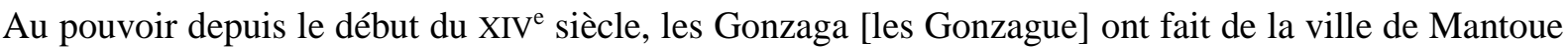
un centre politique et culturel important jusqu'au XVIII ${ }^{\mathrm{e}}$ siècle $^{1}$. Les Gonzague disposent d'un Palazzo Ducale - un palais ducal au centre de la ville - servant de résidence principale à la famille, mais ils possèdent également un grand haras à l'extérieur de la ville. D'après Giorgio Vasari (1550), ce lieu est "situé au milieu d'une prairie où l'on élevait une race de chevaux et de juments". Une écurie y avait été érigée par Francesco Gonzaga - François II de Mantoue - pour abriter des chevaux de race.

En 1524, dans l'esprit de la villa suburbaine réservée aux riches romains de l'Antiquité, Frederico Gonzaga - Frédéric II de Mantoue ${ }^{2}$ - qui avait passé une partie de son enfance à Rome décide de

\footnotetext{
${ }^{1}$ La Maison des Gonzague a fourni à la ville de Mantoue des seigneurs et/ou des podestats à partir du début du XIV ${ }^{\mathrm{e}}$ siècle, puis des marquis du $\mathrm{XV}^{\mathrm{e}}$ au $\mathrm{XVI}^{\mathrm{e}}$, puis des ducs du XVI $\mathrm{X}^{\mathrm{e}}$ au début du XVIII', jusqu'à ce que le duché de Mantoue soit réuni avec le duché de Milan par les autrichiens en 1708.

2 Élevé du titre de marquis à celui de duc en 1530 par l'empereur Charles Quint, Frédéric II de Mantoue (1500-1540) est le fils de François II (1466-1519) et d'Isabelle d'Este (1474-1539). Cette dernière est une grande figure politique et culturelle de la Renaissance qui a développé un mécénat exigeant pour décorer son studiolo au Palazzo Ducale (contenant des œuvres
} 
construire un palais résidentiel à l'écart de la ville - le Palazzo del Te [palais du Té] - dédié au plaisir, à la fête et à la représentation politique, au point qu'il est nommé : la "villa di delizie" [la villa des délices]. Mais le palais est également appelé "il palazzo dei lucidi inganni" [le palais des lucides tromperies] parce qu'il sert les sombres ambitions politiques de Frédéric II qui ira jusqu'à se laisser acheter par Charles Quint pour faire de Mantoue un duché3.

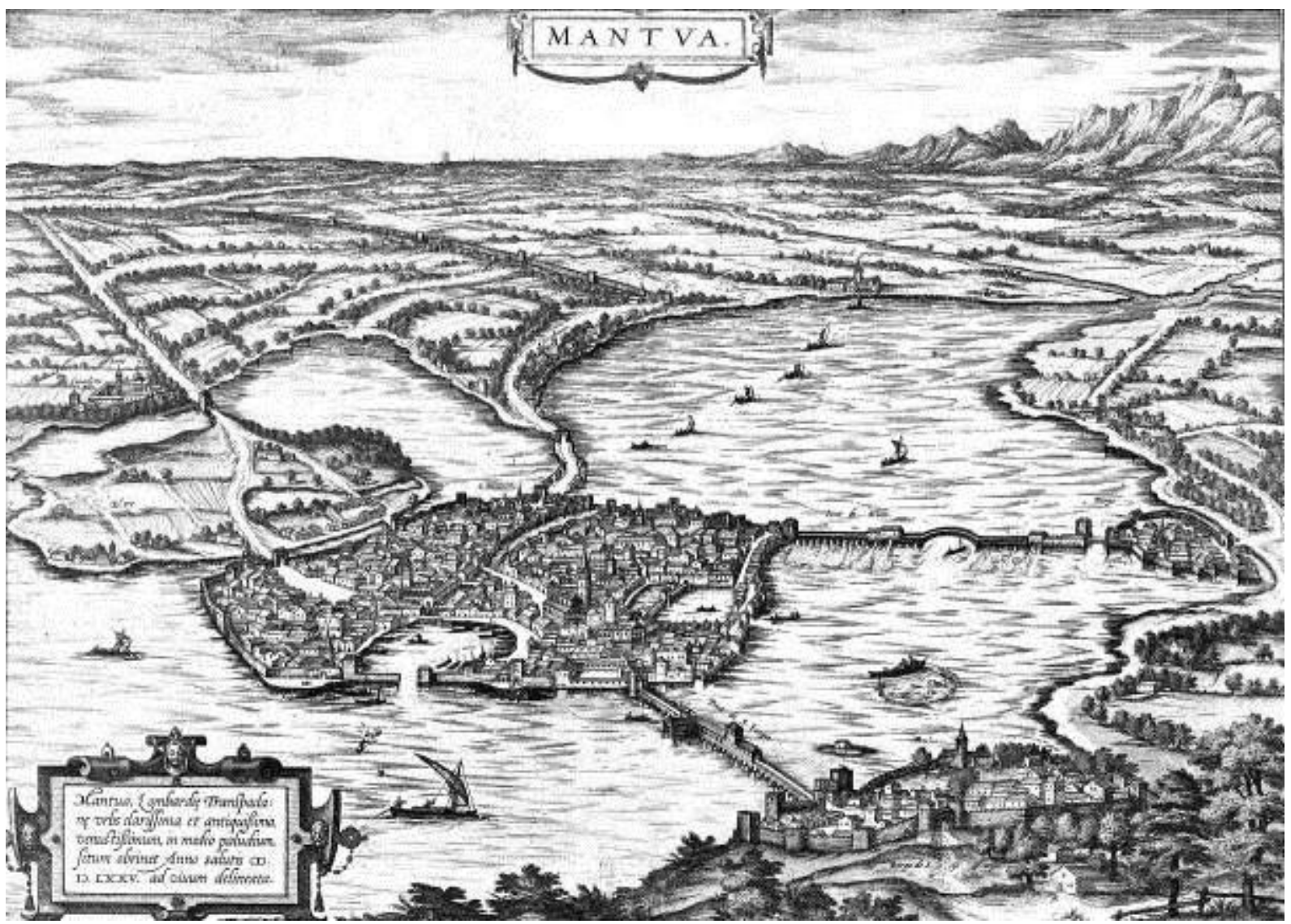

Fig.1 - Georg Braun \& Frans Hogenberg, Mantua, Gravure tirée de Civitates orbis terrarum, 1575, planche 50. L'Isola del Te est située en haut, à gauche, hors des fortifications du centre de Mantoue.

Le palais ne porte pas le nom de Té parce que son plan à la forme de la lettre T, mais parce qu'il était situé au milieu de l'Isola del Te [l'île du Té] ${ }^{4}$. D'après Quatremère de Quincy (1830, p.212), selon

d'Andrea Mantegna, Le Pérugin, Lorenzo Costa, Le Corrège...) et qui a réalisé des commandes à de nombreux artistes prestigieux (Raphäl, Le Titien, Michel-Ange, Léonardo de Vinci...).

3 Les sombres manœuvres politiques de Frédéric II tirent parti du conflit pour la suprématie en Europe entre les Habsbourg menés par Charles Quint - empereur du Saint-Empire romain germanique et roi d'Espagne - et les Valois conduit par François $\mathrm{I}^{\mathrm{er}}$ - roi de France. Alors que son ancêtre Gianfranco Gonzaga (1395-1444) avait obtenu le titre de marquis de l'empereur Sigismond (1368-1437) contre 12000 florins d'or en 1433, Frédéric II espère acquérir le titre de duc en servant Charles Quint. Il a l'ambition personnelle d'extraire Mantoue de l'influence des États pontificaux, ce qui le poussera à trahir la France et les autres petits états italiens, tiraillés entre l'autorité papale et celles des puissances française et espagnole. Son père - François II de Mantoue - avait été un allié de la France, mais sa mère - Isabella d'Este - rompt secrètement en 1522 l'accord qui liait Mantoue aux états pontificaux depuis 1520. En raison de la position stratégique de Mantoue, Charles Quint achète les services de Frédéric II. Participant à la bataille de Pavie de 1525, Frédéric II s'arrange pour y faire emprisonner François I ${ }^{\text {er }}$ par Charles Quint. Il est en contact avec les principaux généraux qui mènent le sac de Rome en 1527- son frère Ferrante dirigeait une faction de l'armée impériale. Enfin, en 1530, il accueille au Palais du Té Charles Quint qui l'élèvera au titre de duc (Hazan, 1986).

${ }^{4} \mathrm{Au} \mathrm{XII}{ }^{\mathrm{e}}$ siècle, en tirant parti de la boucle du fleuve Mincio dans laquelle elle avait été fondée, la ville de Mantoue a organisé un système de défense en créant quatre lacs artificiels (Superiore, Mezzo, Inferiore, Paiolo) ce qui fit ressembler le centre urbain à une île reliée à la campagne par des ponts. Au XVII ${ }^{\mathrm{e}}$ siècle, une forte inondation transforma les lacs en 
"l'opinion d'historiens dignes de confiance", il paraîtrait que "le mot Té fut une abréviation, ou, si l'on veut, une mutilation de tajetto ou tejetto, qui signifiait, dans le langage du pays, coupure ou passage donné à l'écoulement des eaux, et que cette dénomination locale, appliquée au terrain sur lequel le palais fut construit dans la suite, lui aura, par le fait de l'usage vulgaire, communiqué son nom".

À plusieurs reprises, Frédéric II tente de convaincre l'architecte Giulio Romano [Jules Romain], de travailler à son service ${ }^{5}$. Après avoir refusé, Romain finit par s'établir à Mantoue en 1523. En effet, il a été contraint de fuir Rome parce qu'il risquait la prison pour avoir réalisé des dessins obscènes ${ }^{6}$. En 1525, alors qu'il a déjà travaillé pour le Palazzo Ducale, Frédéric II lui confie le projet du palais du Té qu'il construit en à peine dix-huit mois en complétant les bâtiments du haras existant. Son projet tient, à la fois, de la villa romaine et du palais renaissant. L'édifice est très particulier parce que, à la fois, il obéit et il déroge au style du Cinquecento. Lors de la conception, Romain équilibre l'influence du classicisme de son maître Raphaël et celle du maniérisme de Michel-Ange dont il admire le travail. Comme aucune carrière de pierres n'est disponible à proximité du chantier, il utilise des briques recouvertes de stucs ${ }^{7}$. Si bien qu'après la construction rapide du gros-œuvre, il faudra presque dix ans (de 1527 à 1536) pour terminer les travaux de décoration intérieure réalisés par les plâtriers, les sculpteurs et les peintres des fresques.

Le style particulier du palais ne reflète pas seulement la crise de l'institution catholique qui lance la Contre-Réforme - marquée par le passage du classicisme renaissant au baroque -, il est également lié à l'évolution du contexte politique et des ambitions personnelles de Frédéric II qui désire éblouir Charles Quint par le raffinement de sa cour. La finalité de la décoration intérieure du palais est de glorifier la famille régnante des Gonzague et de servir leurs desseins politiques. Dans les décors foisonnants de stucs et de peintures du palais, Romain emploie toutes les ressources de l'illusionnisme et du clair-obscur au service d'allégories dédiées à la gloire (par exemple, le massacre de la salle des Géants) et au plaisir des Gonzague (par exemple, les thèmes érotiques de la salle de Psyché).

Selon l'hypothèse défendue par Olga Hazan (1986), Frédéric II aurait demandé à Romain de représenter par l'architecture et la décoration du palais la fin d'un monde qui s'écroule (celui du Rome de la Renaissance) et le début d'un autre (celui du Mantoue maniériste). Il se sert de l'image de la ruine - du sacrifice rendu nécessaire des romains - pour prétexter une régénérescence - un nouvel âge d'or à Mantoue qui renoue avec le succès de celui de la Rome impériale antique. La renaissance idéalisée à laquelle il aspire justifie les actes sombres à accomplir et lui permet de justifier sa trahison des autres états de la péninsule. En associant Mantoue à la Rome antique, Frédéric II s'impose comme héritier légitime d'un nouveau pouvoir. Par ailleurs, plusieurs indices architectoniques semblent confirmer cette hypothèse :

marécages malsains et le lac Paiolo - au centre duquel se trouvait l'île du Té - fut alors remblayé pour combattre la malaria et étendre la ville.

5 Considérant que Raphä̈l (1483-1520) aurait atteint la perfection en peinture, les artistes du XVI ${ }^{\mathrm{e}}$ siècle développent une nouvelle manière de s'exprimer différente du classicisme renaissant. Parmi eux, l'architecte Giulio Pippi, dit Giulio Romano [Jules Romain] (1499-1546) - surnommé romano parce qu'il est originaire de Rome - a été formé à l'école de Raphaël, mais il a également été fortement influencé par Michel-Ange. À la charnière entre les styles renaissant et baroque, Romain exalte la dimension dramatique de ses œuvres et il développe un système de décoration intérieure, complexe et raffiné, en unissant le stuc et la peinture. Il joue un rôle essentiel dans la diffusion d'un maniérisme anticlassique et grandiloquent.

6 Vers 1523, Romain a réalisé à la plume seize dessins érotiques (peut-être commandés par Frédéric II pour agrémenter l'un de ses cabinets). Le graveur italien de seconde main Marcantonio Raimondi a réalisé des gravures à partir des dessins de Romano qu'il a publié dans I Modi [Les Façons] (1524). La même année, Raimondi sera emprisonné brièvement sous l'ordre du pape Clément viI. L'écrivain italien Pierre l'Arétin créera par la suite les Sonetti lussuriori [Sonnets luxurieux] (1526) inspirées des gravures de Raimondi. L'œuvre finale sera publiée en 1527 juste avant le sac de Rome après lequel Raimondi retrouvera Romain à Mantoue.

7 Alors qu'elle remonte à l'Antiquité, la technique du stuc est remise à la mode par les italiens de la Renaissance (notamment après la redécouverte des fresques de la domus aurea au $\mathrm{XV}^{\mathrm{e}}$ siècle dont le style fantastique et grotesque influencera Raphaël et Michel-Ange, jusqu'à Romain) avant d'être rediffusée dans toute l'Europe. Le stuc est une technique donnant à un matériau plastique l'apparence de la pierre lissée, utilisée pour les murs et les plafonds, en intérieur comme en extérieur. Au palais du Té, Romain utilise un enduit fin chargé de fine poudre de marbre pour un résultat lisse et brillant. 
- depuis les façades extérieures (sud, ouest, nord), entre les pilastres lisses et géants de l'ordre toscan (une forme trapue et simplifiée de l'ordre dorique) les parements des murs sont fabriqués à partir de grands blocs de pierre irréguliers ;

- dans la loggia ouest permettant le passage de l'entrée principale à la cour intérieure, les quatre colonnes sont rustiquées pour parodier le vestibule prestigieux d'un palais romain ;

- dans deux façades intérieures de la cour d'honneur (est, ouest), des triglyphes semblent même tomber de leur frise comme pour signifier la fin d'une époque alors que l'ensemble paraît s'écrouler dans la rustification des façades ;

- dans la façade extérieure donnant sur le jardin (à l'est), le palais semble se reconstituer dans un ordre toscan sophistiqué, impérial et lisse, témoin d'une nouvelle époque.
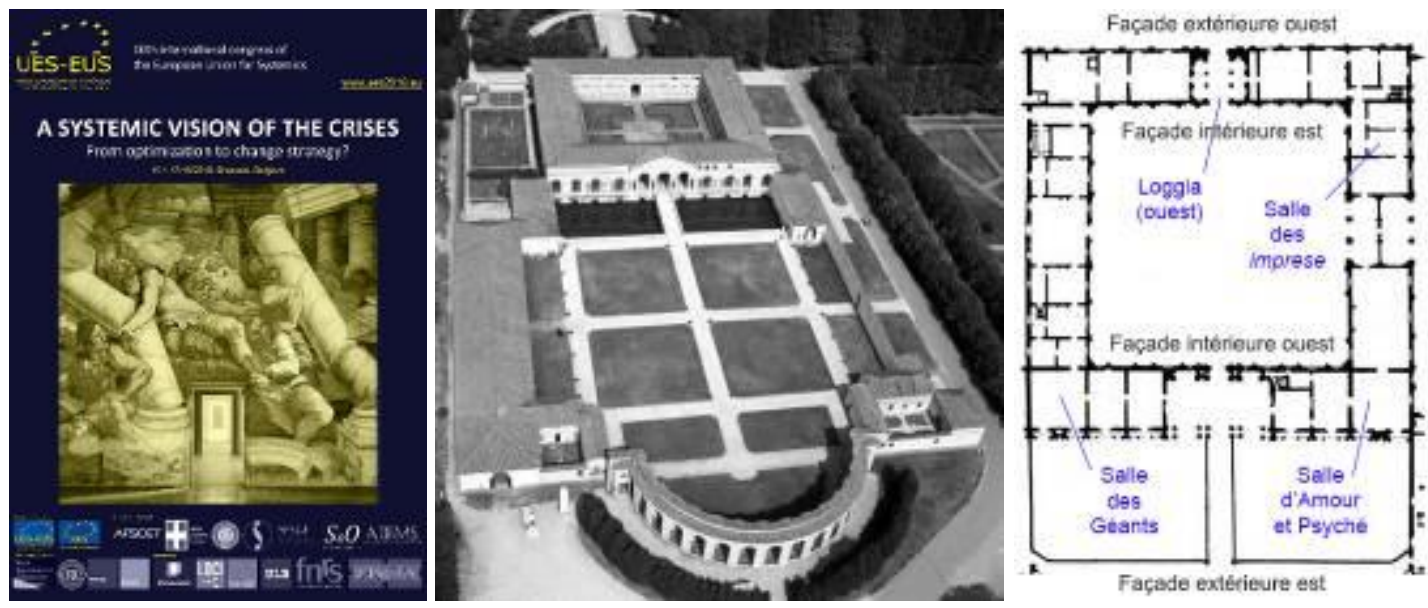

Fig.2 - a. Affiche du congrès international de l'Union européenne de systémique (UES2018). b. Vue aérienne du palais du Té. c. Plan du palais du Té.

\section{INTERMÈDE MYTHOLOGIQUE}

Alors que le contexte d'élaboration de la fresque est partiellement hypothétique, le thème illustré est quant à lui clairement identifiable.

Dans les épisodes mouvementés de la mythologie grecque qui décrivent la généalogie des dieux et la création du monde, deux types de personnages apparaissent : les Titans et les Géants. Les auteurs de l'Antiquité ne les distinguent pas toujours de la même manière et, quand ils le font, les histoires qu'ils racontent à leur sujet varient. Une seule constante narrative traverse ces récits : à la fin de tous les combats qu'ils livrent contre les dieux de l'Olympe, ils sont invariablement vaincus par Zeus ${ }^{8}$.

Ainsi, la Théogonie du poète grec Hésiode (env. VIII ${ }^{\mathrm{e}}-\mathrm{VII}{ }^{\mathrm{e}}$ siècle avant J.-C.) - dont l'existence est incertaine - est un long poème qui raconte - après avoir chanté les Muses en prélude - l'apparition chronologique des dieux depuis l'émergence de Gaïa à partir du Chaos, jusqu'à l'ascension progressive de Zeus au sommet de l'Olympe et la description de sa descendance. La culture de l'époque repose alors sur des muthos (des fables) - où les dieux ne brillent pas par leur moralité - qui permettent de donner sens au lent passage du chaos à l'ordre.

Dans un passage du poème, Hésiode décrit la titanomakhia [la Titanomachie], littéralement le combat contre les Titans ${ }^{9}$. Selon lui, le Chaos - origine première des forces naturelles - engendra Gaïa [la

\footnotetext{
8 Pour bien situer chaque personnage mythologique, il faut être conscient que certains dieux de l'Olympe grecs ont été assimilés par la suite à d'autres dieux du Panthéon romains, avant que les artistes de la Renaissance proposent encore d'autres variations. Les associations suivantes sont établies entre le texte grec d'Hésiode et le texte latin d'Ovide: Ouranos/Uranus, Cronos/Saturne, Zeus/Jupiter, Héra/Junon...

9 "Dans ce jour, un grand combat s'engagea entre tous les dieux et toutes les déesses, entre les Titans et les enfants de Saturne [Cronos] que Jupiter [Zeus] tira des abîmes souterrains de l'Érèbe, pour les rappeler à la lumière, armée formidable, puissante, douée d'une force prodigieuse. Ces guerriers avaient chacun cent bras qui s'élançaient de leurs
} 
terre], qui engendra à son tour de nombreux enfants dont Ouranos (le Ciel) avec lequel elle s'unit pour créer les six Titans (Océan, Cœos, Crios, Hypérion, Japet, Cronos), les six Titanides (Théia, Rhéa, Thémis, Mnémosyne, Phœbé, Téthys), les trois Cyclopes (Brontès, Stéropès, Argès : Géants à un seul œil) et les trois Hécatonchires (Briarée, Cottos, Gygès : Géants qui possèdent cent bras et cinquante têtes). Craignant d'être supplanté par un de ses enfants, Ouranos les enfuit dès leur naissance dans le Tartare (le monde souterrain des Enfers). Mère aimante, Gaïa instruit son fils Cronos pour supprimer Ouranos. Cronos surprend son père endormi, il le castre et il disperse ses parties génitales dans les airs. De la fécondation de Gaïa par le sang coulant de la blessure d'Ouranos naissent les Géants (personnages dont il sera question plus loin), les Érinyes (les furies, déesses vengeresses) et les Méliades (Nymphes des frênes). Au départ, Cronos libère tous ses frères et ses sœurs, il devient le dieu suprême et seul Océan ne le soutient pas. Mais, plus tard, il enferme à nouveau les Cyclopes et les Hécatonchires dans le Tartare et, à son tour, il a peur d'être détrôné, si bien qu'il dévore les enfants que lui donne sa sœur Rhéa. Désespérée, Rhéa demande conseil à Gaïa qui la pousse à soustraire à son père le fils qu'elle porte en elle : Zeus. Si bien que Rhéa accouche secrètement en Crète. Devenu adulte, Zeus veut se venger de son père. Par l'intermédiaire de la Nymphe Métys - fille d'Océan -, Zeus administre à Cronos un breuvage pendant son sommeil et ce dernier vomit tous ses enfants (Poséidon, Hadès, Héra, Déméter, Hestia) avant d'être chassé du ciel par Zeus. Une bataille de dix ans s'engage alors entre le mont Olympe (où règne Zeus et ses frères/sœurs) et le mont Othrys (où se sont réfugiés Cronos et les Titans). Mais les forces surnaturelles des uns et des autres se neutralisent. Rhéa conseille alors à Zeus de libérer du Tartare les Cyclopes et les Hécatonchires, tandis qu'Océan lui conseille de rallier Styx et ses quatre enfants (Zèle, Victoire, Pouvoir, Force). Dès lors, l'équilibre des forces est rompu et les habiles cyclopes offrent : un casque qui rend invisible à Hadès ; un trident qui anime les mers à Poséidon; le tonnerre, la foudre et l'éclair à Zeus. Les puissants Hécatonchires jettent trois cent pierres qui s'écrasent simultanément sur les Titans, tandis que Zeus déclenche un déluge de feu autour d'eux. Le sol se déchire, la poussière s'élève et un bruit effroyable couvre la bataille. Alors qu'ils demandent grâce, Zeus enferme les Titans dans le Tartare et poste en faction les trois Hécatonchires. Enfin, pour venger la défaite des Titans, Gaïa enfanta seule Typhon - un Titan effrayant et surpuissant - que Zeus affrontera et vaincra en combat singulier avant de pouvoir s'installer définitivement au sommet de l'Olympe.

$\mathrm{Au} \mathrm{VI} \mathrm{I}^{\mathrm{e}}$ siècle avant J.-C., alors que les philosophes passent du muthos (mythe) au logos (la raison) en proposant des réflexions morales, politiques et métaphysiques, un autre épisode mythologique est rédigé - la Gigantomachie - et il est moins polémique du point de vue de l'intégrité morale des dieux de l'Olympe.

Ainsi, dans les Métamorphoses (I, 151-162), le poète romain Ovide (I ${ }^{\text {er }}$ siècle après J.-C.) décrit cette seconde guerre ${ }^{10}$ qui aurait éclaté lorsque les douze olympiens (Aphrodite, Apollon, Arès, Artémis, Athéna, Dionysos, Hadès, Héphaïstos, Héra, Hermès, Poséidon, Zeus) auraient définitivement

épaules, et cinquante têtes, attachées à leur dos, planaient sur leurs membres robustes. Opposés aux Titans dans cette guerre désastreuse, tous portaient dans leurs fortes mains d'énormes rochers. [...] Alors Jupiter [Zeus], n'enchaîna plus son courage ; son âme se remplit soudain d'une bouillante ardeur, et il déploya sa force tout entière. S'élançant des hauteurs du ciel et de l'Olympe, il s'avançait armé de feux étincelants ; les foudres, rapidement jetées par sa main vigoureuse, volaient au milieu du tonnerre et des éclairs redoublés et roulaient au loin une divine flamme. [...] Partout se déployait une égale vigueur. La victoire se déclara enfin. Jusqu'alors l'un et l'autre partis, en s'attaquant, avaient montré le même courage dans cette violente bataille ; mais, habiles à soutenir aux premiers rangs un combat acharné, Cottus, Briarée et Gygès, insatiables de carnage, de leurs mains vigoureuses lancèrent coup sur coup trois cents rochers, ombragèrent les Titans d'une nuée de flèches, et, vainqueurs de ces superbes ennemis, les précipitèrent tout chargés de douloureuses chaînes sous les abîmes de la terre" (Hésiode, Théogonie).

10 "Mais les hauteurs de l'éther ne devaient pas être un asile plus sûr que la terre : car les Géants, à ce qu'on assure, voulurent conquérir le royaume des cieux et entassèrent, pour s'élever jusqu'aux astres, montagnes sur montagnes. Alors, le maître tout puissant fracassa l'Olympe sous les traits de la foudre et fit crouler le Pélion à bas de l'Ossa, qui le soutenait. Comme les corps de ces combattants barbares gisaient ensevelis sous une telle masse, leur ouvrage, la Terre [Gaïa], sur qui avait abondement coulé le sang de ses enfants, en fut, dit-on, imbibée ; elle anima ses flots encore chauds et elle en forma, pour ne pas voir finir les derniers rejetons de sa race, d'autres êtres à face humaine. Mais cette race méprisa les dieux ; elle fut, entre toutes, avide des horreurs du carnage et ne respira que la violence ; on reconnaissait qu'elle avait été créée avec du sang." (Ovide, Métamorphoses, livre I, 151-162) 
consolidé leur empire durant la période dégradée de l'âge de fer. Après la défaite des Titans, Gaïa prit progressivement ombrage des nouveaux Olympiens et, en particulier, de son petit-fils Zeus. Elle suggéra aux Géants de lutter contre les nouveaux maîtres du monde ${ }^{11}$. Confiant en leur force et leur taille monstrueuse, les Géants tentèrent de détrôner Zeus pour venger la défaite des Titans. En effet, les Géants voulurent "conquérir le royaume des cieux et entassèrent, pour s'élever jusqu'aux astres, montagnes sur montagnes". Dans la plaine de Phlegra, ils ont tenté d'assaillir l'Olympe en empilant le mont Ossa sur le Mont Pélion ${ }^{12}$. En réponse, Zeus a foudroyé cette masse de rochers, il a repoussé sur terre les Géants et il les a écrasés sous les montagnes qu'ils avaient entassées. Imprégnée de leur sang, Gaïa métamorphosa les Géants en êtres nouveaux, à face humaine, tout aussi violents et impies que les humains de l'âge de fer. La terre est en quelque sorte régénérée par les corps des Géants.
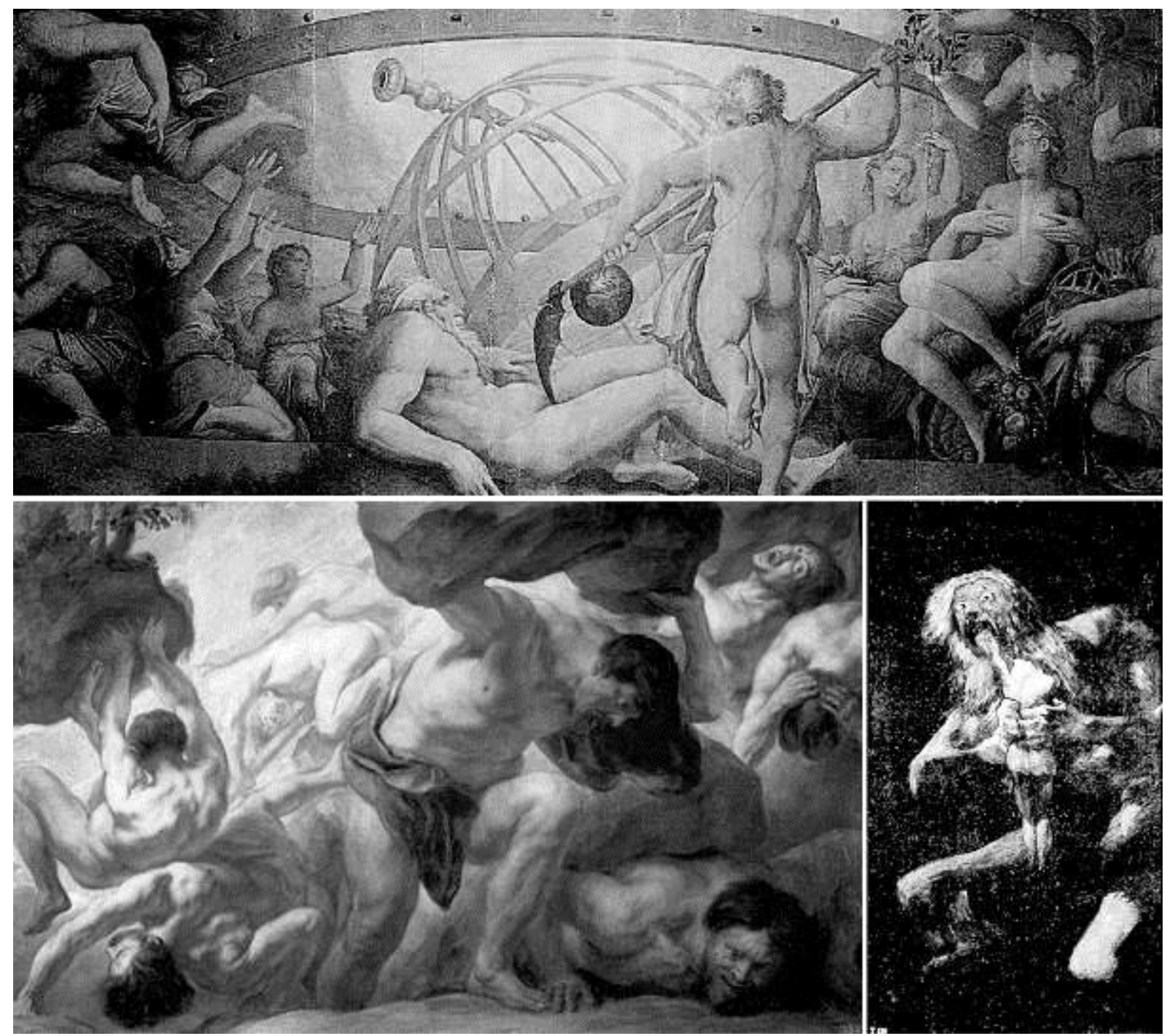

Fig.3 - a. Giorgio Vasari, La mutilation d'Ouranos, Détail de la fresque de la Sala di Cosimo i ${ }^{\text {er }}$ Palazzio Vecchio, Florence, env.1560. b. Francisco de Goya, Saturne [Cronos] dévorant l'un de ses enfants, 1820-1823. c. Jacob Jordaens, La chute des Titans, env.1636-1638.

\footnotetext{
${ }^{11}$ Les Géants sont les enfants de Gaïa, fécondée par le sang d'Ouranos, lorsqu'il fut mutilé par son fils Cronos (Hésiode, Théogonie). Ces non dieux sont souvent représentés comme des monstres anguipèdes et mortels.

${ }^{12}$ Olympe, Pélion et Ossa sont les trois montagnes de Thessalie que les Géants, voulant escalader le ciel, entassèrent l'une sur l'autre, avant d'être foudroyés et écrasés par Zeus.
} 


\section{LA FRESQUE LA CHUTE DE GÉANTS}

Après cette introduction au contexte historique de construction et de décoration du palais du Té et à l'interprétation symbolique de la Gigantomachie, la Caduta dei Giganti [la chute des Géants], peut être analysée.

La fresque est située dans une salle d'angle appelée la Sala dei Giganti [salle de Géants]. Le première a donc donné son nom à la seconde puisque, dans un certain sens, une continuité totale existe entre la tectonique de la salle et la fresque que la décore, puisque toutes les parois intérieures sont peintes sans interruption de la coupole jusqu'au sol : "La Salle des Géants n'a ni début ni fin" (Vasari, 1550) ${ }^{13}$. Contrairement au tableau définit comme "fenêtre ouverte" par Leon Battista Alberti (1435), Romano renforce l'aspect fictif de la peinture qui s'étend partout sauf dans la fenêtre où seul le réel est encadré.

Volontairement mal éclairée, cette salle d'angle surprend le visiteur qui se sent écrasé, englobé, effrayé par la composition peinte sur les parois intérieures. Inspirée d'autres œuvres de l'époque et de l'héritage de Raphaël et Michel-Ange, la fresque est la quintessence de la décoration maniériste ${ }^{14}$ : une image complexe, trouble et sombre, un mélange de vrai et de faux, la rencontre entre moralité du mythe antique représenté, style artistique personnel et ironie débridée. Les corps sont déformés, les formes sont amplifiées et les couleurs sont crues. Dans son œuvre, Romain détourne adroitement certains aspects de la commande de Frédéric II lorsqu'il utilise plusieurs échelles incompatibles pour affirmer l'aspect fictionnel la fresque. Bien qu'écrasé, le spectateur comprend tout de suite que la scène qui l'entoure n'est pas réelle. Les géants sont-ils effrayants ou comiques ? Romano utilise le décalage de l'ironie. Tout est ambigu : un temple minuscule flottant sur quelques nuages résiste au cataclysme, alors que les Géants semblent légers et fragiles.

La fresque reprend le thème de la Gigantomachie au moment où Zeus foudroie les Géants. Au plafond, l'éther est peuplé des dieux de l'Olympe, pressés les uns contre les autres sur une couronne de nuages. Soutenu par d'autres nuages, un temple ionique insensible à la colère de Zeus s'élève tout en haut de l'espace, abritant le trône et l'aigle de ce dernier. Alors qu'ils contemplent les Géants qui s'écroulent sous le poids des pierres et des colonnes, les dieux ont les visages marqués par l'effroi ou la stupeur. Lorsque le visiteur entre dans la salle depuis la salle de l'Empereur, Zeus a quitté son trône et il lui fait face en lançant son faisceau d'éclairs vers la droite. Si le visiteur suit du regard la trajectoire du mouvement, il découvre en contrebas sur le mur ouest le tout début de la chute. Les Géants ont des corps musculeux, démesurés, avec des visages grotesques et désespérés, ils essaient de résister ou d'échapper la pluie de roches et d'éléments architecturaux imaginée par Romain qui "faisait un considérable massacre parmi ses orgueilleux" (Vasari, 1550).

La fresque est essentiellement destinée aux membres de la cour de Mantoue, lettrés, cultivés et capables d'interpréter le sens caché qu'elle contient. L'interprétation la plus probable serait la mise en tension symbolique entre la voluptas et la terror ${ }^{15}$, figurées par deux imprese ${ }^{16}$, est matérialisée par le rapport entre deux salles d'angle du palais (Kliemann \& Rohlmann, 2004) :

\footnotetext{
${ }^{13}$ À l'époque, le sol était couvert d'une mosaïque de galets de rivière peints. Avec l'atténuation des arrêtes du volume obtenue par la peinture, Romain tente d'abolir les parois pour projeter le spectateur dans l'illusion. En plus, la cheminée était utilisée d'abord pour éclairer les peintures plutôt que pour chauffer la pièce.

${ }^{14}$ Pour concevoir la scène de la fresque, Giulio Romano se serait inspiré de différentes œuvres existantes : de l'oculus du plafond de la chambre des Époux (peint par Andréa Mantegna au palais ducal de Mantoue entre 1465 et 1474) pour le petit temple qui couronne la composition; de l'amoncellement des corps de la chapelle Sixtine (peint par Michel-Ange sur le pendentif du serpent d'airain en 1511) pour les nombreux corps écrasés aux visages désespérés; du Concile des Dieux (réalisé par Raphaël et ses élèves au centre du plafond de la loggia de l'Amour et Psyché en 1518 à la villa Farnésina à Rome) pour la ronde des olympiens; du décor de la Bataille du pont Milvius (réalisé par lui-même dans la salle de Constantin du Vatican en 1520 d'après les dessins de Raphaël) pour le paysage derrière les Géants agonisants.

15 Dans le Figino (1591), Gregorio Comanini utilise la fresque de la Salle des Géants comme exemple de l'esthétique de l'horreur: "Je me souviens d'avoir vu à Mantoue, dans une chambre du palais ducal du Té, peints par Jules Romain, les Géants foudroyés à Phlégra, écrasés et brisés par la destruction des rochers et des montagnes, avec des aspects et des mouvements si étranges et si horribles, qui si quelqu'un assistait à un pareil spectacle dans la réalité, il serait sûrement rempli d'horreur et ressentirait un grand tourment à ce spectacle. Et pourtant, comme c'est de l'imitation et de la peinture, il n'est personne qui ne tienne à voir cette œuvre et qui n'en retire un plaisir suprême, comme le montre l'affluence d'étrangers qui y accourent." Dans sa description de la fresque, Giorgio Vasari (1550) affirmait l'effet cathartique de l'œuvre par le
} 
- au nord, la salle de Psyché propose au visiteur un plaisir esthétique - la voluptas - dans des scènes mythologiques érotiques qui valorisent le plaisir de l'âme s'élevant vers les dieux. Dans ce cas, Frédéric II - représenté par l'impresa du lézard - montre qu'il "aime avec chaleur" le sujet loyal ;

- au sud, la salle des Géants expose le visiteur à une frayeur esthétique - la terror - dans une scène où les orgueilleux assailants sont foudroyés et transformés en animaux. Dans ce cas, Frédéric II - représenté par l'impresa du mont Olympe - "interdit la révolte" et "punit avec autorité" le sujet déloyal qui tenterait de l'attaquer.

Dans l'esthétique de l'horreur de la salle des Géants, de nombreux symboles exprimeraient le mouvement de régénérescence de Mantoue et le changement d'alliances politiques sans altération du pouvoir des Gonzague :

- Zeus foudroyant les Géants semble personifier Charles Quint imposant son pouvoir sur l'Italie - les princes italiens et la papauté - en châtiant les impies de Rome aux dépens des français ${ }^{17}$. L'aigle sur le trône de Zeus symboliserait les armes des Habsbourg, alors que la foudre figurerait le pouvoir impérial ;

- la stabilité de l'Olympe et du temple divin qu'il contient, hors d'atteinte des quatre vents, de la foudre et de l'amas de pierres qui s'écroule célèbre le caractère immuable du pouvoir des Gonzague et montre à tous que toute révolte contre l'ordre est impossible et insensée ;

- la terre formée par les corps des Géants devient des montagnes et des vallées ce qui symbolise la régénérescence, la destruction de l'ancien monde romain permettant l'émergence d'une nouvelle ère pour Mantoue ;

- par l'association de l'aigle et du mont Olympe, la fresque montrerait la fidélité de Frédéric II à Charles Quint et légitimerait ainsi le pouvoir des Gonzague.

"naturel" de l'imitation illusionniste mêlant réel et fiction. Par contre, distinguant la peinture "qui feint les choses qui n'existent pas" en reproduisant le réel de "celle qui imite les choses qui sont" en produisant une fiction, Comanini prolonge l'hypothèse aristotélicienne de la Poétique en affirmant que cette fresque est une fiction qui provoque chez le spectateur un effet cathartique bien plus puissant que ne pourrait l'avoir une œuvre réaliste, parce qu'elle joue sur le plaisir esthétique d'être confronté viscéralement à une vision d'horreur tout en sachant qu'il ne s'agit pas du réel.

16 À la différence de l'emblème contenant trois éléments (la devise, la figure, l'épigramme), l'impresa - pratique spécifique à l'Italie du XVI ${ }^{\mathrm{e}}$ siècle - contient deux éléments : le motto [la devise, le mot] ou l'anima [l'âme] et la figura [la figure] ou le corpo [le corps] pour exprimer une concetto [une idée]. Une impresa qualifie symboliquement le programme moral du porteur à qui elle est attribuée (par exemple un noble) en associant un motto et une figura, de manière énigmatique. Une impresa lie une devise volontairement succincte sensée révélé un trait saillant de la personnalité du porteur (un idéal, une vertu, un événement personnel) à une figure (objet, plante, animal...). Ainsi, dans la Camera della Imprese du palais du Té, Frédéric II est représenté par au moins deux imprese : l'impresa des Gonzague montrant la figure du mont Olympe associée à la devise fides [foi] (en référence à la foi, qui doit être forte à la fois chez le prince et chez ses sujets) et son impresa personnelle montrant la figure du lézard (ou la salamandre) associée à la devise Quod huic deest me torquet [Ce qui lui manque me tourmente]. Charles Quint est quant à lui représenté par la figure de l'aigle. Dans le palais du Té, le mont Olympe, le lézard et l'aigle sont représentés de nombreuses fois.

${ }^{17}$ Sur le passage de l'empereur Charles Quint d'autres œuvres d'art ont été exécutées pour le mettre en scène dans l'attitude du grand sauveur de la patrie capable d'unifier toutes les parties du royaume italien. Souverain catholique, Charles Quint avait annoncé qu'il lutterait contre les protestants dans son empire. Il désapprouva donc officiellement le sac de Rome perpétré par ses soldats (pour la majorité des allemands luthériens décidés à se venger de la papauté), alors qu'il l'avait indirectement ordonné et qu'il acceptait la soumission du pape (il lui aurait cédé 400000 ducats et plusieurs cités italiennes). Pour renforcer la propagande positive dont il a fait l'objet, certains ont considéré le sac de Rome comme une bavure alors qu'il y avait eu des milliers de victimes. 

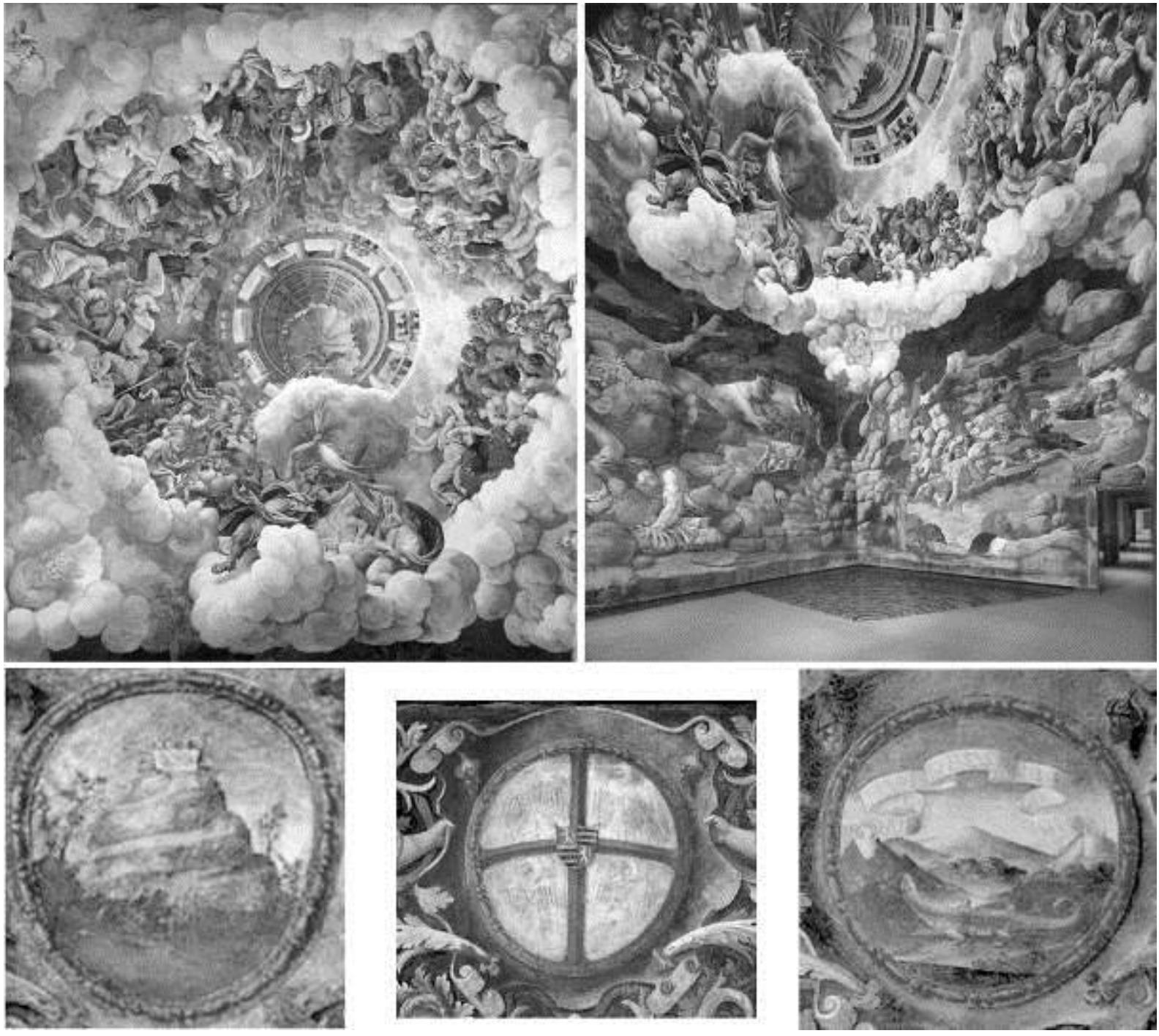

Fig.4 - a. Jules Romain, La Chute des géants, Fresque du plafond, palais du Té, Mantoue, Italie, 1532-1534. b. Médaillons peints, Camera delle Imprese, palais du Té, Mantoue, Italie, 1524 : le mont Olympe (parois est, $2^{\mathrm{e}}$ médaillon à partir de la gauche), les armes des Gonzague (parois sud, médaillon du milieu) et le lézard (parois sud, médaillon de droite).

\section{DE LA LIMITE DE CROISSANCE AU SEUIL D'ÉMERGENCE}

Comment établir un lien entre le récit mythique de La chute des Géants et la théorie des systèmes pour éclairer des situations de crise contemporaines?

Contrairement au mythe assimilable à une forme d'explication du monde fondée sur une construction imaginaire - notamment par le biais de la personnification de certains phénomènes -, la théorie des systèmes est une méthode de modélisation qui tente de comprendre les dynamiques sous-jacentes aux interactions entre, à la fois, les membres de la société, la société et l'environnement éco-systémique.

Établir des liens entre mythe et science n'est pas si étonnant. Selon l'anthropologue français Claude Lévi-Strauss (1962), le bricolage à l'œuvre dans la pensée mythique est comparable à l'ingéniosité de l'invention technique moderne. La "pensée sauvage" n'aurait donc rien à envier à la pensée scientifique. En effet, les récits mythiques et les théories scientifiques sont autant de stratégies développées par l'humanité pour s'assurer une assise existentielle dans un univers dont la connaissance réelle dépasse la somme des capacités cognitives de ses membres. À défaut de connaître totalement le 
réel, l'être humain tente de l'appréhender de manière répétée pour pouvoir y mener des actions et ces tentatives donnent naissance à des réalités multiples ${ }^{18}$.

Le récit mythique semble entrer en résonance avec la fin du monde annoncée par des scientifiques et largement relayée par les médias. À l'image des Géants écrasés sous une pluie de pierre, les crises écologique, sociale et politique qui secouent notre monde contemporain seraient autant de symptomes de l'effondrement probable de notre civilisation et du déclin de nos sociétés. Et la cause première de la crise multiforme actuelle serait d'abord l'inadéquation entre le mode de développement de l'espèce humaine et la rareté relative des ressources disponibles sur terre qui ne permetrait plus à l'humanité de subvenir à ses besoins ${ }^{19}$.

Mais comment en sommes-nous arrivés à enviseager l'effondrement imminent de notre civilisation?

Présent dès le début de l'époque moderne et développé parallèlement à l'émergence du cartésiannisme au $\mathrm{XVII}^{\mathrm{e}}$ siècle $^{20}$, le concept de croissance associé à l'idée du progrès est au cœur des idéologies occidentales ayant piloté le $\mathrm{XX}^{\mathrm{e}}$ siècle et guidant encore largement les actions menées en ce début du $\mathrm{XXI}^{\mathrm{e}}$ siècle. L'histoire idéalisée de l'origine mythique de l'espèce humaine est celle d'un processus de croissance - le progrès - dans lequel un être vivant ontologiquement vulnérable s'extrait progressivement de la pression d'une phúsis hostile grâce au développement de la technè et du politikos (Claeys, 2018). Dans tous les cas, le paradigme de la croissance n'est encore que marginalement questionné. Aborder la question de la limite à la croissance relève d'un défi tant ce paradigme fonde actuellement les stratégies, les décisions, les espoirs et même le langage, à tous les niveaux de la société.

Pourtant, le concept de limite a été largement étudié avec une approche systémique dans l'historique et controversé Rapport Meadows, diffusé dans The Limits to Growth: A report for The Club of Rome's project on the predicament of mankind (Meadows, Meadows, Randers, Behrens III, 1972), traduit en français par Halte à la croissance?, avant d'être réactualisé récemment dans The Limits to Growth: the 30-Year Update (Meadows, Meadows, Randers, 2004) ${ }^{21}$. Ainsi, dans de Limits to Growth, les

${ }^{18}$ Comme déjà démontré à plusieurs reprises, la distinction entre le réel et les réalités est inhérente à la condition humaine et l' "homo doublement sapiens" (re)configure constamment un "réel augmenté" (Claeys, 2018). Le réel augmenté est "un double du réel co-construit par l'homme - qualifié d'augmenté - contre lequel, impertinent, le réel résiste dès qu'il est provoqué. Il possède plusieurs partitions - qui ne font pas partie du réel - telles que les réalités perçue, conçue, vécue, mythique, objective, subjective, intersubjective..." (Claeys, 2013). Contrairement à la réalité augmentée qui nécessite des moyens techniques pour surimposer des informations au réel perçu, le réel augmenté est produit directement par notre conscience.

${ }^{19}$ L'être humain est "équipé initialement d'un inné insuffisant pour survivre naturellement et affecté d'une connaissance limitée du réel malgré l'acquis de ses expériences". Comme tout système vivant, il est d'abord "inachevé" parce qu'il a "des besoins physiologiques fondamentaux" dont "la satisfaction est nécessaire pour sa survie" et dont "la plupart nécessitent des actions dans le réel" (oxygène, eau, nourriture, température corporelle constante, élimination, logement, repos, reproduction). Mais lorsque ces besoins premiers sont assouvis, l'être humain est également "incomplet" parce qu'il a "des besoins psychologiques et sociaux fondamentaux", nécessaires pour contrebalancer l'angoisse existentielle liée à la coupure du réel opérée par le langage rythmant ses états de conscience (Claeys, 2018).

${ }^{20}$ En analysant l'état de l'art de nombreuses hypothèses scientifiques sur le sujet par le prisme des exigences formelles géologiques, Simon L. Lewis et Mark A. Maslin (2015) ont fixé l'année 1610 comme début possible d'une nouvelle époque géologique appelée l'anthropocène - l'âge de l'être humain - parce qu'elle correspond à un minimum historique du taux de $\mathrm{CO}_{2}$ atmosphérique, dans une période jusqu'alors inédite d'homogénéisation du biote terrestre, également appelée l' "échange colombien" ou le "grand échange" par la mise en contact entre l'Ancien et le Nouveau Monde initiée en 1492. Par ailleurs, René Descartes (1637) écrit que la pratique de la science - la méthode analytique - peut "nous rendre comme maîtres et possesseurs de la nature". La nature est ainsi transformée en objet de consommation et elle doit être dominée pour répondre aux besoins humains. Alors que Georges L.L. Buffon (1778) écrit que "la face entière de la Terre porte aujourd'hui l'empreinte de la puissance de l'homme", Charles R. Darwin (1859) montre que l'évolution des espèces repose sur le principe de la sélection naturelle, ce qui sous-entend que seules les espèces les mieux adaptées à leur environnement survivent. Ce qui est une "valorisation (in)directe de l'espèce humaine qui, biologiquement inadaptée, aurait surclassé toutes les autres espèces" (Claeys, 2018).

${ }^{21}$ L'américain Jay W. Forrester est un théoricien de la dynamique des systèmes et un pionnier en modélisation informatique. Après avoir travaillé notamment sur Urban Dynamics (1969), il tente une réponse aux questions soulevées par le Club de Rome - un groupe informel et international d'hommes d'affaires, de dirigeants et de scientifiques - sur les équilibres globaux en ébauchant un modèle dynamique de base : le World1. Ensuite, il documente le modèle final - World2 - dans Worlds Dynamics (1971) qui traite de la modélisation des interactions complexes dans les sphères économique, 
auteurs publient l'analyse prospective de douze scénarios à partir de la modélisation dynamique des systèmes basée sur le modèle World3 de Jay W. Forrester en montrant "différents modes de développement de l'humanité sur deux siècles", entre 1900 et 2100. Ce modèle tient compte de différentes dimensions environnementales, sociétales et économiques. À l'époque, le rapport avait alerté l'humanité sur les conséquences à long terme d'une économie fondée sur le principe de la croissance exponentielle - illustré par des boucles de rétroactions positives - sans que rien ne vienne contrebalancer ce mouvement global. Les questions traitées par ce groupe dans les années 1970 étaient par exemple: "les politiques actuelles nous conduisent-elles vers un avenir soutenable ou vers l'effondrement? Que peut-on faire pour créer une économie humaine qui fournisse de tout en quantité suffisante à tous ?". Le rapport remettait donc en cause le mythe de la croissance économique sans fin. Mais malheureusement en ce début $\mathrm{du} \mathrm{XXI}^{\mathrm{e}}$ siècle, alors que la situation écologique et sociale de l'humanité semble critique, ces questions restent en suspens !

Corollairement à l'idée que toute croissance peut avoir une limite, le concept de décroissance a progressivement émergé au moins depuis les années 1970, notamment lorsqu'il a été progressivement popularisé après la réunion et de la traduction de textes de Nicholas Georgescu-Roegen (qui n'emploie pas le mot) par Jacques Grinevald et Ivo Rens dans Demain la décroissance. Entropie, écologie, économie (1979).

Dans Le principe responsabilité (1979, p.15), Hans Jonas part du constat que "Le Prométhée définitivement déchaîné, auquel la science confère des forces encore jamais connues et l'économie son impulsion effrénée, réclame une éthique qui, par des entraves librement consenties, empêche le pouvoir de l'homme de devenir une malédiction pour lui". Il affirme que l'espèce a le pouvoir technologique de s'autodétruire et ce pouvoir est aux mains de quelques individus ayant la responsabilité de l'humanité entière. Parallèlement à un pouvoir plus grand, de nouveaux devoirs apparaissent pour l'être humain. Selon lui, l'éthique traditionnelle reposait sur l'évaluation des actions (par exemple, mentir, c'est $\mathrm{mal}$ ), alors qu'aujourd'hui elle devrait porter sur l'évaluation de la conséquence des actions (par exemple, moins consommer est bien pour anticiper l'épuisement futur des ressources planétaires). Le concept de responsabilité résume ce devoir moral nouvellement construit par l'intégration de la question de l'avenir (la conséquence des actes) et de la planète entière (la conséquence sur l'écosystème global de ces mêmes actes). Il va jusqu'à écrire que les êtres humains doivent questionner l' "impératif inconditionnel de préserver leur existence pour l'avenir". L'être humain doit donc adapter son projet téléologique pour qu'il réponde à des principes éthiques traditionnels, mais également à d'autres qui ne sont plus uniquement anthropocentriques à partir du moment où ils intègrent la phúsis. À une plus grande autonomie de l'être humain grâce à la technologie correspondrait donc une plus grande responsabilité.

À la même époque, au cours de la formalisation de la pensée systémique destinée à modéliser des systèmes ouverts (von Bertalanffy, 1968) - impossibles à modéliser à l'aide de théories déterministes et mécanistes -, le concept d'autonomie est devenu central dans l'étude des systèmes vivants, humains et sociaux à partir du moment où les systémiciens les ont étudiés en intégrant leurs capacités à s'autoorganiser. De là, Humberto R. Maturana \& Francisco J. Varela (1972) ont défini le concept d'autopoïese issu des deux mots grecs autos (soi) et poiein (produire). L' "auto-production" est donc l'aptitude et la propriété fondamentale de tout organisme vivant de s'auto-produire, c'est-à-dire de produire sans cesse sa propre existence, et de s' "auto-réguler". Ils développent la théorie des systèmes "auto-poïétiques", à la fois, fermés et ouverts. En effet, ils sont fermés au niveau fonctionnel (ils maintiennent leur "identité" pour maintenir leur "clôture opérationnelle"), alors qu'ils restent ouverts au niveau structurel grâce à l'action constante des boucles de rétroaction qui les constituent (le système complexifie ses composants ou produit dans/par ses interactions avec son environnement de

démographique et environnementale. Enfin, il confie le travail de développement d'un troisième modèle - le World3 -, en développant la structure et en enrichissant quantitativement la base de données, à Donella H. et Denis L. Meadows au sein du Groupe Dynamique des Systèmes de la Sloan School of Management du Massachusetts Institute of Technology (MIT) entre 1970 et 1972. Ces modélisations servent de matrice au Rapport Meadows (1972) qui prévoyait un effondrement dans 50 ou 100 ans en cas de croissance exponentielle non maîtrisée des sociétés humaines et de leur consommation. Les prévisions sont correctes de 1972 à 2002. 
nouvelles configurations, il intégre de nouveaux composants depuis l'environnement ou ils supprime certains de ses composants). Pour s' "auto-organiser", d'un côté, le système fait évoluer sa "structure" et, de l'autre, son "comportement". L'autonomie d'un système vivant ne signifie donc pas ici qu'il possède une liberté totale d'action.

À partir d'études de systèmes institutionalisés dans des domaines différents (école, médecine, transports, énergie...), Ivan Illich établit dans ses écrits un lien entre les concepts d'autonomie et de seuil pour développer une théorie de la contre-productivité. Selon lui, "lorsque l'institution se développe et dépasse une certaine limite ou un certain seuil, elle devient nuisible et fortement dysfonctionnelle par rapport à ses propres objectifs et finalités. L'institution devient alors contreproduction, contre-productivité. Elle aliène l'être humain et détruit la société globale" (Assogha, 1979). Autrement dit : "Lorsqu'une activité outillée dépasse un seuil défini par l'échelle ad hoc, elle se retourne d'abord contre sa fin, puis menace de destruction le corps social tout entier." (Illich, 1973). Selon Illich, des seuils identifiables sont donc capables de faire basculer tout système institutionalisé de l'autonomie vers l'hétéronomie et la dépendance. Dans la société moderne, il associe ces seuils à un déséquilibre entre l'usage d'outils conviviaux (maniables, accessibles à tous) et celui d'outils manipulables qui reposent sur des technologies maîtrisée par des spécialistes et dont la fabrication relève d'un processus industriel. C'est ainsi qu'il préconise un débat public sur l'établissement de seuils.
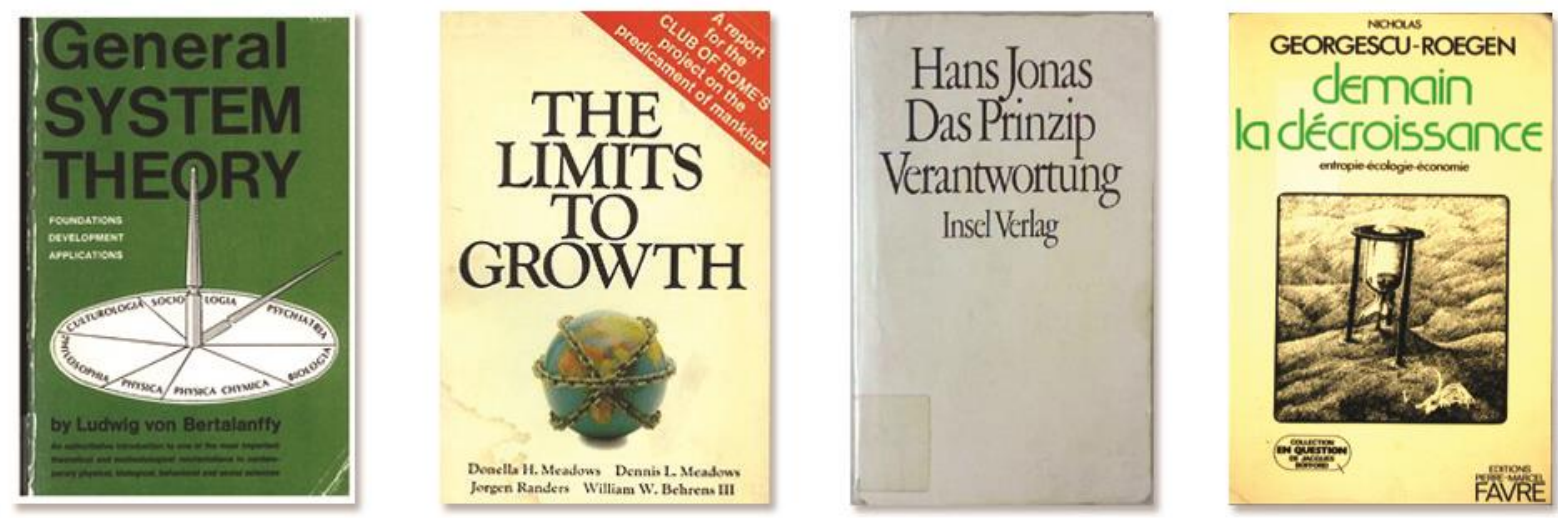

Fig.5 - Premières de couvertures emblématiques

(Bertalanffy, 1968 ; Rapport Meadows, 1972 ; Jonas, 1973 ; Georgescu-Roegan, 1979).

En théorie des systèmes, le concept de seuil est lié à celui d'émergence.

Dans leurs liens avec le concept d'émergence, les effets de seuil ont été notamment mis en lumière par le biologiste américain Stuart A. Kauffman (1995) qui étudie l'origine de la vie avec la théorie de la complexité et des systèmes auto-organisés. Il a imaginé un jeu avec des boutons et des fils pour faire comprendre son modèle théorique. Pour démarrer le jeu, des milliers de boutons percés ansi que des fils ont été placés de manière aléatoire sur un tapis. Au cours d'une période de temps déterminée, le joueur répète plusieurs fois l'opération suivante : il glisse des fils dans les trous des boutons et puis il tire sur l'un ou l'autre des fils pour voir combien de boutons se déplacent avec le fil. Au début du jeu, le joueur qui tire sur un fil n'entraîne qu'un petit nombre de boutons. Mais, progressivement, plus le joueur tire de fils plus ceux-ci vont s'emmêler et attirer plus de boutons. Au point que des petits groupes de boutons commenceront à s'entremêler et à former des groupes de plus en plus grands : des "clusters". À partir des données du jeu, une courbe peut être tracée dans un repère cartésien ( $c f$. figure 6) avec, en abscisse, le ratio fils/boutons (le nombre de fils divisé par le nombre de boutons) et, en ordonnée, le cluster potentiellement le plus grand (la taille du plus gros cluster susceptible d'être mis en mouvement lorsque le joueur tire un fil). La courbe est presque horizontale au début, puis elle se redresse subitement lorsque le ratio fils/boutons se situe aux alentours de 0,5 (quand $50 \%$ des boutons sont interconnectés) et, enfin, elle se stabilise à nouveau pour être proche de l'horizontale. À partir de ce ratio, même si le joueur continue à ajouter des fils, ses chances d'attraper un plus grand groupe de boutons que le précédent diminuent puisque presque tous les boutons sont reliés. 


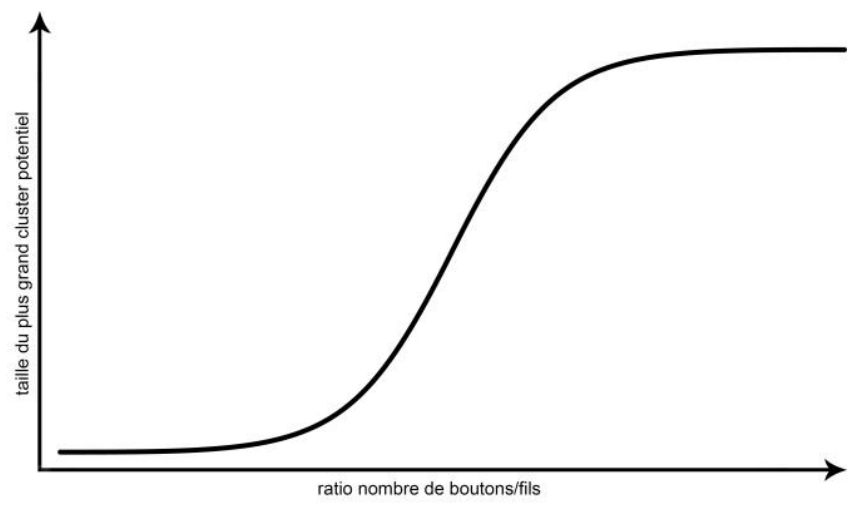

Fig.6 - Courbe en S caractéristique d'une transition de phase d'après les effets de seuil à l'œuvre dans le jeu des boutons et des fils de Stuart A. Kauffman (1995).

La courbe en $S$ de Kauffman est une fonction sigmoïde apparaissant de manière similaire dans de nombreux champs disciplinaires (physique, biologie, logistique, art...). L'allure de cette courbe est caractéristique de l'apparition brutale de seuils lorsque de petits éléments ou des clusters se connectent en réseaux à l'intérieur de systèmes complexes : elle illustre le phénomène de la transition de phase. Lorsque certaines conditions extérieures au système changent brutalement ou de façon discontinue, ses propriétés changent également puisque les valeurs des variables externes permettant sa modélisation évoluent. Alors le système subit une transition de phase, c'est-à-dire une transformation remarquable d'un état vers un autre comme, par exemple, quand l'eau se transforme en glace. Dans le modèle de Kauffman, les boutons et les fils peuvent être remplacés par des personnes ou des processus. De manière générale, ce jeu montre que les propriétés individuelles des éléments pris isolément ne définissent pas celles du système complexe qu'ils forment. Ce sont les relations qui définissent le système et ce sont elles qu'il faut observer pour le comprendre. À partir d'un seuil - un niveau critique de connectivité - tout ensemble de composants isolés devient un réseau dynamique et complexe.

Le concept de seuil par la description de la transition de phase est donc directement lié au concept d'émergence, autrement dit au fait que "le tout est différent des parties" : "Certaines propriétés du tout apparaissent spontanément et ne peuvent être déduites directement à partir des parties et de leurs relations de base, mais résultent néanmoins de leurs interactions", ce qui "contraste avec une approche uniquement hiérarchique des parties" (Claeys, 2013). Développé porgressivement à partir de l'émergentisme britannique de la fin du XIX ${ }^{\mathrm{e}}$ siècle, le concept d'émergence est définissable à partir de trois principes (Claeys, 2013) :

1. la non-additivité : l'émergence apparaît sous l'effet d'une cause complexe différente de la somme des effets provoqués par des causes partielles isolées ;

2. la nouveauté : l'émergence est une croissance continue de complexité parallèle à un processus créatif ;

3. la non-déductibilité : une propriété émergente est impossible à déduire de façon logique, même avec une connaissance la plus complète possible des propriétés du phénomène observé, bien que ce soit parfois seulement révélateur des limites de l'état des connaissances au moment de l'observation.

Un exemple canonique du phénomène d'émergence dans un système naturel donné par le philosophe britannique Georges H. Lewes (1874) est celui de l'eau : "Une solution à base de deux corps gazeux simple, deux atomes d'hydrogène $(H)$ et un atome d'oxygène $(O)$, permet l'émergence d'un corps liquide unique : l'eau $\left(\mathrm{H}_{2} \mathrm{O}\right)$. Dans des conditions standard de température et de pression, le tout (l'eau) possède alors au moins une propriété (elle est liquide) que ne possédaient pas ses parties constituantes (les atomes d'hydrogène et d'oxygènes)." (Claeys, 2013) Un autre exemple canonique du phénomène d'émergence - cette-fois dans un système social - est celui de la fourmilière géante des fourmis coupeuses de feuille: "La reine ne donne pas d'ordres directs, et chaque fourmis réagit individuellement et spontanément aux odeurs chimiques et laisse à son tour un parfum pour les autres 
fourmis. C'est un comportement récursif, qui résulte de la structure ordonnée des mouvements, de la construction, de la recherche et de la disposition à un niveau macroéconomique. [...] La forme de la fourmilière émerge de la relation entre les fourmis et le contexte, cette forme n'était pas prévisible avant." (Claeys, 2013).

En requestionnant la crise contemporaine à l'aide des concepts systémiques d'auto-organisation et de seuil plutôt que d'utiliser ceux de croissance et de limite, les enjeux du Rapport Meadows peuvent être élargis. En effet, ce rapport tout comme de nombreuses démarches écologistes mettent en évidence la relation problématique entre l'humanité et l'environnement, entre un système ouvert et son contexte. La limite à la croissance est rendue nécessaire par la rareté des ressources naturelles disponibles. La durabilité repose sur l'utilisation des ressources présentes dans l'environnement et la capacité de cet environnement à les renouveler. Les limites étudiés sont alors celles imposées par la nature et de sa capacité à permettre l'activité humaine. Le contexte limite la croissance du système ouvert à partir du moment où les échanges entre eux ne sont plus possibles.

D'un point de vue philosophique cette approche revient à s'inscrire dans le principe de la séparation de l'humanité et de son environnement, de l'opposition traditionnelle entre culture et nature. Cette séparation conceptuelle a été largement remise en cause aujourd'hui chez de nombreux auteurs. Ainsi, dans Par-delà nature et culture (2005), l'anthropologue français Philippe Descola entend dépasser le dualisme qui oppose nature et culture en montrant que la nature est elle-même une production sociale. Selon lui, l'opposition nature/culture - héritage de la méthode cartésienne - ne fait aujourd'hui plus sens parce qu'elle relève d'une pure convention sociale rendue aujourd'hui caduque. Il développe une anthropologie dite non dualiste, en ce sens qu'elle ne sépare pas en deux domaines ontologiques distincts les humains et les non-humains. Elle s'intéresse aux relations entre humains et non-humains autant qu'à celles entre humains. De là, il propose la constitution de ce qu'il nomme une "écologie des relations".

Or la crise contemporaine ne se situent pas uniquement dans le rapport de l'humanité à son environnement, mais s'inscrit à l'intérieur même du système humain : des règles qui en régissent son fonctionnement, des frontières entre espace privés, public et communs. Les crises envisagées ne sont alors pas dues à l'accident et la contingence lié à l'environnement mais sont endogènes, propres au fonctionnement du système humain. Outre le fait que le contexte limite la croissance du système ouvert, tout en gardant son identité fonctionnelle, ce dernier - auto-organisé - doit évoluer structurellement pour permettre les échanges avec le contexte sous peine de limiter également sa croissance.

En passant de la modélisation d'un système ouvert - mais structurellement figé - à partir de la limite de la croissance à la modélisation d'un dysfonctionnement inhérent au système, certaines propositions de solution, répandues dans les discours actuels semblent moins pertinentes comme moyen d'éviter ou de sortir de la crise (par exemple : le développement technologique va nous sauver, les espoirs d'une énergie inépuisable, l'exploitation des ressources dans l'espace...).

Une hypothèse alternative pour sortir de la crise pourrait être envisagée en développant la capacité des communautés humaines d'établir des seuils à ne pas dépasser afin de préserver l'autonomie de ses sous-systèmes, capable de développer et protéger ses communs. Cette communauté aurait alors un rapport à son environnement tel que la question de l'exploitation problématique des ressources ne se poserait de manière différente. Certes, ce point de vue nous rend pleinement responsable des choix à faire. La responsabilité impliquent que nous sommes acteurs et que la fatalité n'est pas de mise. Le défi est de taille car outre le paradigme de la croissance, d'autres croyances ou mythes représentent des freins (l'individualisme, les théories des besoins, l'antagonisme binaire entre privé et public...).

À propos du commun, le philosophe Pierre Dardot et le sociologue Christian Laval (2014) soulignent judicieusement le défi comme étant inhérent au système et non comme un rapport entre humanité et nature : "le commun ne relève ni de l'essence des hommes, ni de la nature des choses, mais de l'activité des hommes eux-mêmes : seule une pratique de mise en commun peut décider de ce qui est 'commun', réserver certaines choses à l'usage commun, produire les règles capables d'obliger les hommes". 


\section{CONCLUSIONS : LES LIMITES DE LA CROISSANCE : UN TABOU ?}

Finalement, pourquoi susciter un rapport entre La chute des Géants et la thématique du congrès UES2018? Est-ce un clin d'œil à l'art italien sachant que le congrès était une initiative de l'AIRS ${ }^{22}$ ? Est-ce parce qu'elle montre l'effondrement d'éléments architecturaux, alors que le congrès avait lieu dans une faculté d'architecture ${ }^{23}$ ? Non. Le choix s'est porté sur cette fresque parce qu'elle possède une force suggestive opérante à plusieurs niveaux et, plus encore, parce qu'elle questionne de manière originale le concept multiforme de crise.

En entrant dans la salle des Géants par la porte du mur nord, le spectateur est immergé au cœur de l'effondrement d'un monde en crise. Mais, en pleine expérience cathartique, lorsque le spectateur tourne dans l'espace, il refait face à la porte par laquelle il est entré ( $c f$. figure 7). Elle semble réduite par rapport aux dimensions exagérées des personnages fictifs qui périssent sur le mur peint. Mais elle est réelle! Avec elle, le spectateur prend conscience de l'illusion dans laquelle le plonge cette esthétique de l'horreur. À la manière du point de fuite d'une perspective linéaire, la porte agit sur les sens du spectateur en lui offrant - alors que la salle est finie - un point à l'infini au centre de l'horizon perspectif. Par analogie, notre horizon immédiat est la limite de ce que nous pouvons observer à partir de la situation que nous occupons actuellement. Notre horizon semble occupé par le processus d'effondrement de notre monde. Aujourd'hui, l'utopie est de croire que tout continuera comme avant, notre horizon est celui de l'effondrement. Mais l'effondrement est également la régénerescence désirée par Frédéric II de Mantoue en le mettant en scène. Bien qu'il semble indépassable au moment où nous l'observons, cet horizon est modifiable avec le temps. À travers, le spectacle effroyable des Géants écrasés par les pierres saturant notre perception, la petite porte pourait être le point de départ d'un nouvel espace socio-écologique à déployer. Chaque fois que nous faisons un pas, cet horizon évolue en fonction de notre compréhension du monde qui nous entoure.

Si la limite est par définition inatteignable, le seuil renvoie au point de basculement au-delà duquel émerge du neuf dans un sens évolutif ou destructeur. Derrière l'horizon saturé de misère sociale, de déchets et d'informations d'un XXI ${ }^{\mathrm{e}}$ siècle aux prises avec la "silicolonisation du monde" (Sadin, 2016), une issue matérialisée par la porte semble offrir une possibilité de dépassement. Non pas une nouvelle relance de la croissance en équilibrant dans l'urgence les relations dynamiques entre le système humain et son écosystème, mais une voie potentielle de mutation intrinsèque du système humain, associée à la définition de seuil révisable de croissance. En effet, l'auto-organisation n'est ni une liberté sans limite, ni une gestion autarcique, elle combine une structure ouverte et une fonction fermée. L'autonomie est la capacité de l'être humain a fixer des seuils pour gérer les interactions dynamiques entre l'environnement, la société et l'individu, de fixer des besoins fondamentaux de base révisables. La crise dans sa forme la plus sévère signifie pour l'humanité : la mort, le désordre, l'entropie. Or, le propre du vivant et de l'être humain est sa capacité à l'auto-organisation ou autonomie. Un système est autonome s'il est capable d'établir ses propres règles, de les faire évoluer en fonction du contexte et de les suivre.

Une acception courante de l'autonomie l'assimile à la liberté et à l'indépendance. Cependant, nous faisons l'hypothèse, à la suite d'Illich, que ce qui caractérise et rend possible l'autonomie, c'est avant tout l'acceptation et le choix de limites auto-imposées à soi-même ou au système, puisqu' "il nous faut déterminer avec précision ces échelles et les seuils qui permettent de circonscrire le champ de la survie humaine" (Illich, 1973). En effet, dans notre vie quotidienne, l'utilisation d'outils dont nous ne contrôlons pas le fonctionnement ne favorise pas l'autonomie. Ainsi, l'approvisionnement en énergie, la dépendance à l'industrie agroalimentaire ou le traitement numérique de nos informations personnelles sont des domaines dans lesquels nous renonçons - au moins une partie - à notre autonomie. Le choix de l'autonomie peut se réaliser à différents niveaux :

\footnotetext{
${ }^{22}$ Le congrès a été initié par l'Associazione Italiana per la Ricerca sui Sistemi (AIRS).

${ }^{23}$ Le congrès a eu lieu à la Faculté d'architecture, d'ingénierie architecturale, d'urbanisme (LOCI) de l'Université catholique de Louvain (UCLouvain), à Bruxelles, les 15, 16 et 17 octobre 2018.
} 
- au niveau micro, le citoyen peut utiliser des outils et des technologies qu'il peut maîtriser, plutôt que d'utiliser ceux nécessitant une maîtrise uniquement accessible au spécialiste, il peut choisir de se réapproprier la gestion de certains besoins qu'il avait abandonné à des prestataires de services, il peut privilégier l'utilisation de produits non-transformés par l'industrie...;

- au niveau méso, la communauté locale peut favoriser les coopérations et garantir un accès équitable aux biens communs ;

- au niveau macro, les pouvoirs publics peuvent rester attentif à la contre-productivité pouvant les affecter, ils peuvent mettre en débat les seuils dans l'utilisation des ressources, ils peuvent réguler la protection et la restauration des biens communs, ils peuvent faciliter la réappropriation par le citoyen d'espaces d'actions et de décisions démocratiques, ils peuvent inclure le citoyen dans des processus d'évaluation de projets.

L'horizon n'est pas une simple limite perceptive, à la fois statique et prévisible, comme un miroir reflétant de manière autoréférentielle nos aspirations. Nous projetons un horizon et l'horizon nous modifie en retour. Avoir un horizon ne nous donne pas la certitude d'obtenir les effets auxquels nous aspirons. Néanmoins, toute communauté de chercheurs a besoin d'un horizon commun pour se mettre en marche ${ }^{24}$, pour se mettre en projet, provoquer une émulation, faire évoluer les savoirs et les connaissances. Confrontés à l'hypothèse d'un effondrement socio-écologique, quel seraient l'horizon et le rôle de la communauté des systémiciens pour aider notre société en crise ?

Les développements de la pensée systémique pourraient aider la société à :

- considérer sérieusement le niveau local comme une ressource : soutenir les initiatives locales comme des leviers de transition socio-écologique. Sur le terrain, de nombreux actions et projets se développent en jouant de manière créative avec la limite à la croissance (multiples initiatives citoyennes, formes de management favorisant l'autonomie, pédagogies novatrices dans le domaine de l'éducation...) ;

- valoriser la frugalité potentielle de notre existence : toutes ces initiatives locales impliquent une certaine sobriété dans l'usage des ressources et visent l'autonomie (alimentaire, énergétique, scientifique...). Elles s'inscrivent dans un temps long en privilégiant le commun (mise en relation, consolidation du lien, négociation des règles...);

- améliorer la connaissance du niveau méso entre niveau macro et niveau micro : développer des approches plus créatives, plus originales pour établir des liens entre local et global ;

- augmenter la compréhension des systèmes socio-écologiques complexes : tout en gardant une distance critique par rapport à nos modèles théoriques, créer de nouveaux outils pratiques et théoriques d'aide à la décision pour éclairer les pouvoirs publics.

Actuellement, le défi principal de notre communauté n'est peut être plus le développement théorique de la science systémique. L'enjeu sera plutôt d'incarner encore plus dans le réel cette théorie pour quelle devienne une pensée vivante permettant une représentation différente du monde, pour qu'elle puisse s'épanouir en tant que langage d'interprétation des expériences vécues (pratique) et en tant que représentation d'un avenir désirable en soi (esthétique). Est-ce la chute des Géants nécessaire à une régénérescence ? Est-ce une crise à l'horizon de nos méthodes scientifiques avant l'émergence d'un nouveau monde?

\footnotetext{
${ }^{24}$ Marcheur, il n'y a pas de chemin, Le chemin se construit en marchant." D'après le poème d'Antonio Machado (1936, p.205), Proverbios y cantarès, Campos de Castilla, écrit en 1917 et régulièrement cité dans la littérature systémique.
} 


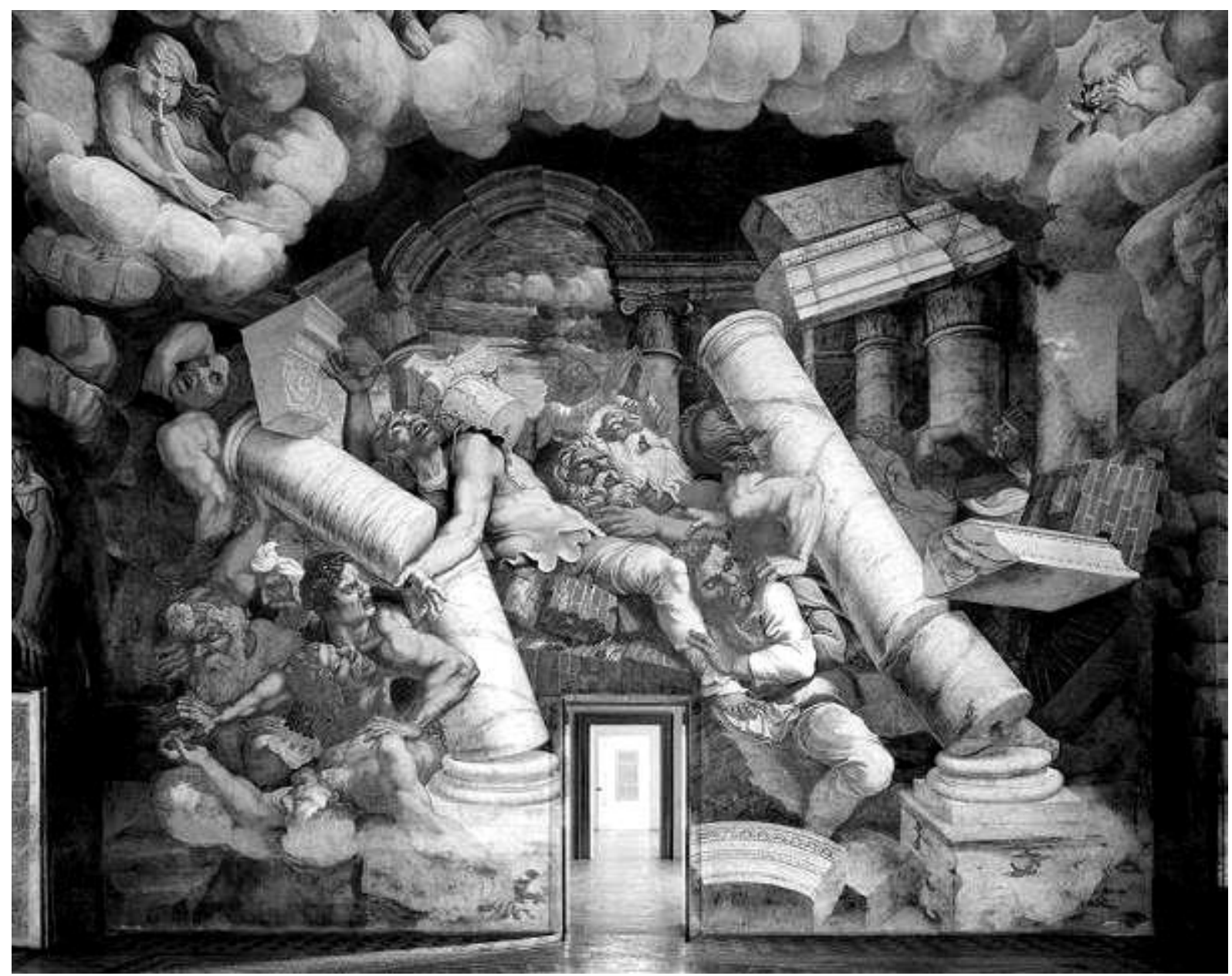

Fig.7 - Jules Romain, La Chute des géants, Fresque du mur nord de la salle des Géants, palais du Té, Mantoue, Italie, 1532-1534.

\section{RÉFÉRENCES}

ALBERTI, Leon B. (1435). La peinture. Trad. Thomas Golsenne \& Bertrand Prévost, rev. Yves Hersant [De pictura, éd.1436]. Paris : Seuil (coll. Sources du savoir).

ASSOGHA, Yao (1979). "Ivan Illich : Essai de synthèse". Critères, Montréal, n²6, pp.217-235.

BERTALANFFY, Ludwig (von) (1968). Théorie générale des systèmes. Trad. Chabrol J.-B. [General System Theory. New York: Georges Braziller]. Paris : Dunod, éd.1993.

BRAUN, Georg, NOVELLANUS, Simon \& HOGENBERG, Frans (1575). Civitates orbis terrarum volume ii. Cologne. Voir également Stephan Füssel (dir.) (2017). Villes du monde : Édition intégrale des planches coloriées 1572-1617. Taschen : Cologne.

BRAUN, Georg \& HOGENBERG, Frans (1572-1617). Civitates orbis terrarum [Les cités de la terre]. Cologne, vol.2.

CLAEYS, Damien (2018). "Pour une co-conception écosystémique de l'architecture à l'ère de l'anthropocène". Roose, Marie-Clotilde (dir.) (2019). Penser à partir de l'architecture : Poétique, technique, éthique. Louvain-la-Neuve : Presses universitaires de Louvain.

CLAEYS, Damien (2013). Architecture \& complexité: Un modèle systémique du processus de (co)conception qui vise l'architecture. Thèse de doctorat de l'Université catholique de Louvain. Louvain-la-Neuve : Presses universitaires de Louvain.

Collectif (2015). Le grand atlas de la mythologie : Gréco-romaine et égyptienne. Paris : Atlas. 
COMANINI, Gregorio (1591). Figino ou de la finalité de la peinture. Trad. Marie-France Courriol [Il Figino overo del fine della Pittura, Mantoue], Paris : Hermann, éd.2014.

DARDOT, Pierre \& LAVAL, Christian (2014). Commun : Essai sur la révolution au XXI siècle. Paris : La Découverte

DESCOLA, Philippe (2005). Par-delà nature et culture. Paris: Gallimard (coll. Folio Essais), éd.2015.

FORRESTER, Jay W. (1971). World Dynamics. Cambridge, MA: Wright-Allen Press.

FORRESTER, Jay W. (1969). Urban Dynamics. Waltham, MA: Pegasus Communications.

GEORGESCU-ROEGEN, Nicholas (1979). Demain la décroissance: Entropie, écologie, économie. Trad. Jacques Grinevald \& Ivo Rens. Lausanne : Pierre-Marcel Favre.

HAZAN, Olga (1986). Giulio Romano et Frederico Gonzagua au Palazzo Te. Mémoire présenté à l'Université du Québec à Montréal comme exigence partielle de la maîtrise en études des arts, 231pp.

HÉSIODE. Théogonie. Trad. Anne Bignan [Aimé-Martin, M. L. (dir.), Ernest Falconnet (éd.) (1838). Les petits poèmes grecs... Paris : Auguste Desrez, pp.127-139].

ILLICH, Ivan (1973). La Convivialité. Paris : Seuil.

JONAS, Hans (1973). Le principe responsabilité : Une éthique pour la civilisation technologique. Trad. Jean Greisch [Das princip verantwortung]. Paris : Flammarion (coll. Champs), éd.2013.

KAUFFMAN, Stuart A. (1995). At Home in the Universe: The Search for Laws of Self-organization and Complexity. Oxford: Oxford University Press.

KLIEMANN, Julian \& ROHLMANN, Michael (2004). Fresques Italiennes du XVI siècle: De Michel-Ange aux Carrache. Trad. Aude Virey-Wallon[Wandmalerei in Italien: Hochrenaissance und Manierismus 1510-1600]. Paris : Citadelles \& Mazenod.

LEWES, Georges H. (1874). Problems of Life and Mind: First series: The Foundations of a Creed. London: Trübner.

MATURANA, Humberto R. \& VARELA, Francisco J. (1972). "Autopoiesis: The Organization of the Living". Trad. Maturana H.R. [De Máquinas y Seres Vivos]. Autopoiesis and Cognition: The Realization of the Living. Dordrecht: Kluwer Academic Publishers, éd.1980, pp.59-138.

MEADOWS, Donella H., MEADOWS, Dennis L., RANDERS, Jørgen (2004). Les limites à la croissance (dans un monde fini) : Le Rapport Meadows, 30 ans après. Trad. Agnès El Kaïm [The Limits to Growth: the 30-Year Update. New York: Universe Books]. Paris : Fayard.

MEADOWS, Donella H., MEADOWS, Dennis L., RANDERS, Jørgen \& BEHRENS III, W. W. (1972). Halte à la croissance? Trad. Jacques Delaunay [The Limits to Growth: A report for The Club of Rome's project on the predicament of mankind. New York: Universe Books]. Paris : Fayard.

OVIDE. "Les Géants". Métamorphoses, livre I, 151-162 [Trad. G.T. Villenave, Paris, 1806].

QUATREMÈRE de QUINCY, Antoine Chrysostome (1830). Histoire de la vie et des ouvrages des plus célèbres architectes du XI jusqu'à la fin du XVIII siècle, accompagnée de la vue du plus remarquable édifice de chacun d'eux. Paris : Jules Renouard, 1830, tome I.

SADIN, Éric (2016). La silicolonisation $d u$ monde: L'irrésistible expansion du libéralisme numérique. Montreuil : L'échappée (coll. Pour en finir avec).

VASARI, Giorgio $(1550 ; 1568)$. La vie des meilleurs peintres, sculpteurs et architectes. Trad. André Chastel (dir.). Paris : Berger-Levrault (coll. Arts), éd.1984, vol.7, pp.163-202. 